\title{
Numerical Analysis of Cylindrical Shell under Axial Impact
}

\author{
WX Zhang ${ }^{1,2 *}$ \\ ${ }^{1}$ Nantong Polytechic College, Nantong, Jiangsu, 226002, China \\ ${ }^{2}$ School of Civil and Environmental Engineering, Hunan University of Science and Engineering, Yongzhou, Hunan, 425199, China
}

\begin{abstract}
The shell mainly resists the external load by the mid plane stress distributed uniformly along the thickness, rather than the bending stress varying along the thickness. Compared with the traditional flat plate, the shell can make full use of the material strength, so it has greater bearing capacity. In water conservancy projects, shells are widely used, such as double curvature flat shell gate, arch dam and so on. Thin shell theory is a classical theory in shell.
\end{abstract}

\section{Introduction}

Thin shell theory is based on the assumption of straight normal $[1,2]$. These assumptions include: (1) the normal strain perpendicular to the middle plane is extremely small and negligible. (2) The normal of the middle plane is kept as a straight line, and the right angle between the normal of the middle plane and its vertical segment remains unchanged, that is, the shear strain in the two directions is zero. (3) The normal stress on the section parallel to the middle plane is far less than that on the vertical plane, so its effect on deformation can be neglected. According to the theory of elasticity, the basic equation of thin shell can be established by using the above hypothesis. They include: the equilibrium equation of the surface in the shell, the geometric equation, the physical equation and various boundary conditions on the boundary.

The allowable disturbance is determined by the statistics and probability distribution of the environment in which the structure is located, and the required safety

$$
\Pi_{k}=\frac{1}{2} D\left(\kappa_{x}^{2}+\kappa_{\theta}^{2}+2 v \kappa_{x} \kappa_{\theta}+2(1-v) \kappa_{x \theta}^{2}\right)+\frac{N_{x}}{2}\left(\partial_{x} w\right)^{2}
$$

and

$$
\Pi_{t}=\frac{1}{2} \rho h\left(\frac{\partial u}{\partial t}\right)^{2}+\frac{1}{2} \rho h\left(\frac{\partial v}{\partial t}\right)^{2}+\frac{1}{2} \rho h\left(\frac{\partial w}{\partial t}\right)^{2}
$$

So the Lagrange function, the Hamiltonian action and the dynamic boundary conditions are

$$
L=\iint\left[\frac{\rho h}{2}\left(\partial_{t} u\right)^{2}-\frac{E h}{2}\left(\partial_{x} u\right)^{2}-\frac{E h}{2 r^{2}} w^{2}+\frac{2(1-v)}{r^{2}}\left(\partial_{x} \partial_{\theta} w\right)^{2}-\frac{N_{x}}{2}\left(\partial_{x} w\right)^{2}\right] r d \theta d x
$$




$$
N_{x}= \begin{cases}-N & 0 \leq x \leq c t, t \leq l / c \\ 0 & c t<x \leq l, t \leq l / c \\ -N & 0<x \leq 2 l-c t, t>l / c \\ -2 N & 2 l-c t<x \leq l, t>l / c\end{cases}
$$

and

$$
\left\{\begin{array}{l}
\left.w \cdot\left[D \partial_{x}^{3} w+D \frac{(1-v)}{r^{2}} \partial_{x} \partial_{\theta}^{2} w+N_{x} \partial_{x} w\right]\right|_{x=x_{0}}=-\left.w \cdot Q_{x}\right|_{x=x_{0}}=0 \\
\left.\partial_{x} w \cdot D\left(\partial_{x}^{2} w+\frac{v}{r^{2}} \partial_{\theta}^{2} w\right)\right|_{x=x_{0}}=-\left.\varphi_{x} \cdot M_{x}\right|_{x=x_{0}}=0
\end{array}\right.
$$

For the two cases of free support, fixed support and simple support, the above formula can be rewritten as follows:

$$
\begin{aligned}
& \left\{\begin{array}{l}
u=\frac{v}{r^{2}} \partial_{r}^{2} w \\
w=0 \\
M_{x}=D\left(\partial_{x}^{2} w+\frac{v}{r^{2}} \partial_{\theta}^{2} w\right)=0
\end{array} \quad\left(x=x_{0}\right)\right. \\
& \left\{\begin{array}{l}
w=\frac{(1-v)}{r^{2}} \partial_{x} \partial_{\theta}^{2} w \\
Q_{x}=D \partial_{x}^{3} w+N_{x} \partial_{x} w=0 \\
M_{x}=D\left(\partial_{x}^{2} w+\frac{v}{r^{2}} \partial_{\theta}^{2} w\right)=0
\end{array} \quad\left(x=x_{0}\right)\right.
\end{aligned}
$$

and

$$
\left\{\begin{array}{l}
Q_{x}=D \partial_{x}^{3} w=0 \\
w=0 \\
\phi_{x}=\partial_{x} w=0
\end{array} \quad\left(x=x_{0}\right)\right.
$$

\section{Numerical example}

Fig. 1 and Fig. 2 describe the external pressure of the first and second order. It can be seen from the figure that the critical load decreases with the increase of external pressure. For the case of only external pressure, buckling can also occur under axial tension impact. 


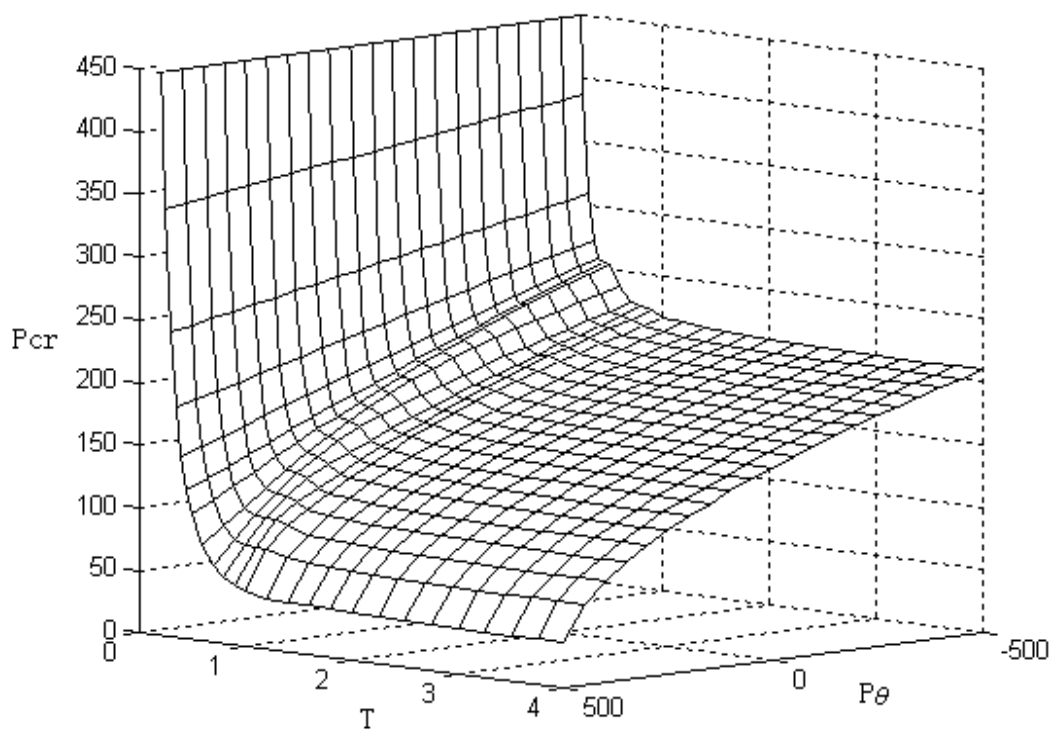

Fig 1. External pressure of the first order

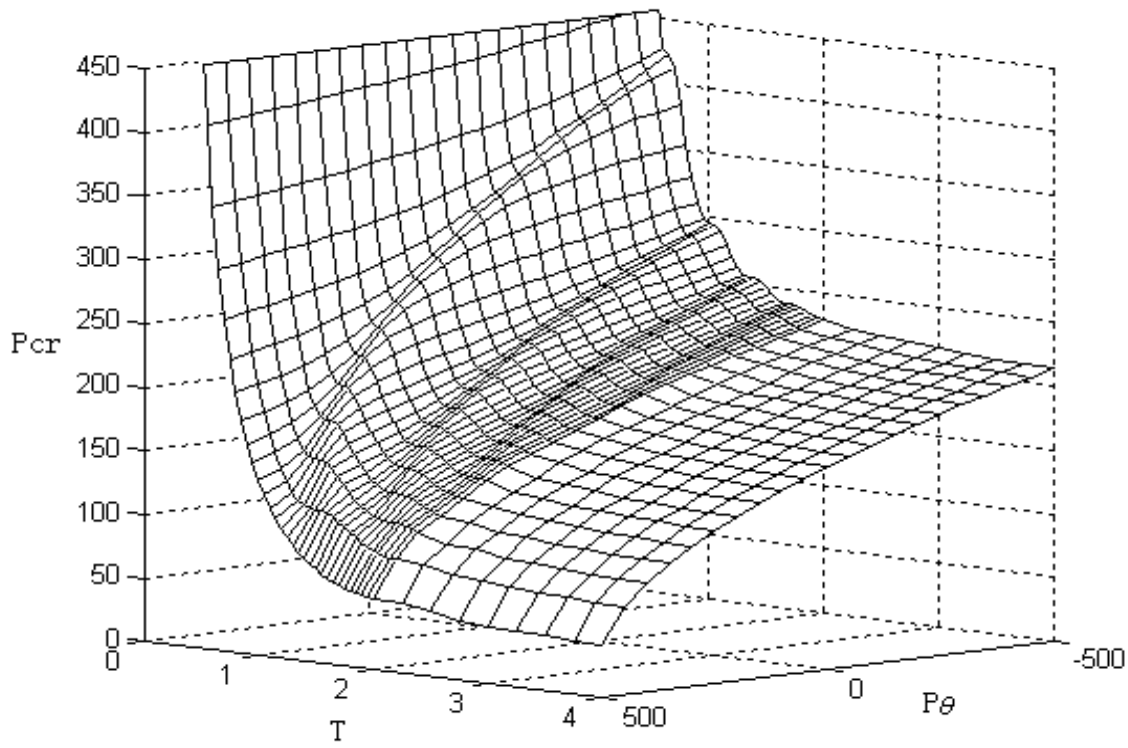

Fig 2. External pressure of the second order

\section{Conclusion}

The dynamic stability of a structure is defined as the dynamic buckling of a structural system in dynamic equilibrium, which is considered as the critical dynamic load if the motion reaches the specified limit within the required service life of the structural system after receiving a small allowable disturbance.

\section{Reference}

1. Huang, D.T. (2013) Effects of constraint, circular cutout and in-plane loading on vibration of rectangular plates. Int. J. Eng. Sci., 68: 114-124.

2. Sahoo, R., Singh, B.N. (2013) A new shear deformation theory for the static analysis of laminated composite and sandwich plates. Int. J. Eng. Sci., 75: 324-336.

3. Chen, G.Q., Wu, Z., Zeng, L. (2012) Environmental dispersion in a two-layer wetland: Analytical solution by method of concentration moments. Int. J. Eng. Sci., 51: 272-291. 
4. Zhong, Y.F., Qing, W.Z., Yu, W.B., Yang, W., Jiao, L. (2015) A variational asymptotic theory of composite laminated plates: Hybrid transformation to Reissner-Mindlin model. Int. J. Eng. Sci., 97: 113125.

5. Comninou, M. (1977) The interface crack. J. Appl. Mech. Trans. ASME 44: 631-636.

6. Govorukha, V., Kamlah, M. (2004) Asymptotic fifields in the fifinite element analysis of electrically permeable interface cracks in piezoelectric bimaterials. Arch. Appl. Mech. 74: 92-101.

7. Herrmann, K.P., Loboda, V.V. (2000) Fracturemechanical assessment of electrically permeable interface cracks in piezoelectric bimaterials by consideration of various contact zone models. Arch. Appl. Mech., 70: 127-143.

8. Bergstrom, J.S., Boyce, M.C. (1998) Constitutive modeling of the large strain time-dependent behavior of elastomers. J. Mech. Phys. Solids, 46: 931-954. 